RESEÑA 



\section{Velázquez, Guillermo Ángel (Dir.) (2016). Geografía y Calidad de vida en Argentina. Análisis regional y departamental (2010).}

Tandil: UNICEN, $351 \mathrm{pp}$.

NÉSTOR JAVIER GÓMEZ

Facultad de Humandiades y Ciencias, Universidad Nacional del Litoral.

Consejo Nacional de Investigaciones Científicas y Tecnológicas.

Es creciente en la Argentina, en los últimos años, el desarrollo de líneas de investigación que adoptan como eje central la cuestión de las condiciones de vida y la calidad de vida de la población. Tal auge es reconocible tanto en mesas temáticas de reuniones científicas como en artículos de revistas especializadas y asimismo en libros, que se dedican a presentar el resultado de investigaciones o bien discusiones conceptuales acerca del abordaje de las condiciones de vida de los habitantes de los territorios. Particularmente, estas líneas de investigación han cobrado notoria fertilidad dentro del campo disciplinar de la geografía, dando lugar a estudios de calidad de vida que hacen hincapié en el eje territorial.

Allí es donde puede ubicarse el libro objeto de esta reseña, dado que en él converge una serie de trabajos emanados de una pluralidad de autores argentinos, en su gran mayoría geógrafos, radicados en diferentes centros de investigación del país, otorgando una mirada «federal» a la cuestión estudiada.

El libro se titula Geografía y calidad devida en Argentina. Análisis regional $y$ departamental (2010), y se trata de una compilación de trabajos dirigida por Guillermo Velázquez y del cual participan 24 docentes-investigadores, en calidad de autores. El libro da cuenta del potente desarrollo de estudios en torno a la calidad de vida de la población o que tienen a la calidad de vida como eje de estudio y discusión. Lo cual contribuye a otorgar dinamicidad al ámbito de la investigación geográfica argentina.

La pluralidad de recortes temáticos utilizados en los trabajos, como también las escalas de análisis, áreas de estudio, fuentes de información, encuadres teóricos, marcos regionales y contextos ambientales, constituyen una de las mayores riquezas de esta compilación. 
El proceso de diferenciación territorial en términos de la calidad de vida de la población se relaciona con un entramado de factores explicativos subyacentes, entre los cuales son destacados las etapas de desarrollo económico de la Argentina, la evolución y características de la población, los cambios en el sistema urbano, la dinámica socioterritorial del espacio rural o la dimensión ambiental. Editado por la Universidad Nacional del Centro de la Provincia de Buenos Aires en el año 20I6, este libro se organiza en cinco partes y dentro de estas contiene 22 capítulos más las reflexiones finales, la bibliografía, el anexo estadístico y una referencia a los autores.

La primera parte, la «Introducción», expone el propósito del libro, que es actualizar contribuciones anteriores respecto del conocimiento de la diferenciación social, económica, ambiental y regional de la calidad de vida de la población argentina, con el mayor nivel de análisis espacial posible, para intentar establecer el peso de diferentes factores explicativos. A su vez, en este apartado se realiza una recopilación histórica de estudios geográficos argentinos relacionados con la calidad de vida. Entre otros, se enumeran capítulos de la obra La Argentina. Suma de Geografia (Aparicio y Difrieri 1958-1963) y también el estudio El país de los argentinos (Chiozza 1975-1977), una obra de seis volúmenes con un nítido enfoque regional, entre otros.

La segunda parte, titulada «El proceso de diferenciación territorial», consta de cinco capítulos que apuntan a poner en evidencia la impronta territorial de procesos económicos, sociales y demográficos, haciendo hincapié la dinámica de los sistemas urbanos y rurales. Aquí escriben Fernando Manzano, Guillermo Velázquez, Adela Tisnés, Santiago Linares, Josefina Di Nucci, Claudia Mikkelsen, Sofia Ares y Matías Gordziejczuk. Los principales argumentos tratan sobre las condiciones de vida de la población argentina en relación a las diferentes etapas de la historia económica y social. Asimismo, se focaliza en la evolución temporal de la población argentina desde el Primer Censo Nacional (I869), incorporándose las estimaciones más antiguas (Censo Vértiz) e incluso otras previas de siglos anteriores. Se exponen gráficos de volúmenes de población, tasas de crecimiento medio anual (por mil), evolución d ella población, tasas de crecimiento vegetativo, porcentajes de población extranjera limítrofe y no limítrofe, saldos migratorios, tasas globales de fecundidad, tasas de nupcialidad y de mortalidad, entre otros. Dado que se incorporan datos desagregados por provincias, se 
afirma que hacia 2010 en cuanto a la esperanza de vida, se mantiene la «polarización entre el norte argentino (menor) y el centro y el sur del país (mayor)». En el mismo sentido, el norte argentino, acusa las mayores tasas de mortalidad por enfermedades infecciosas. En el Capítulo 5 se establecen relaciones entre las dinámicas demográficas y económicas para el período 1947-2010, dando cuenta del comportamiento entre las migraciones internas y el producto bruto geográfico de las jurisdicciones provinciales, señalándose que más que corregir la situación de desigualdad existente, se ha incrementado. En el Capítulo 6 se presenta un pormenorizado análisis de los cambios en el sistema urbano argentino operados entre 1947 y 20I0, aportando las tendencias más recientes desde 200I, como el importante crecimiento y dinamismo de las ATIs menores. Respecto de la dinámica demográfica del espacio rural argentino -planteada en el Capítulo 7-, se observan notorios contrastes. Si bien es clara la tendencia a la reducción de la población residente de modo disperso en el espacio rural, luego existen áreas con clara revitalización poblacional y otras con decrecimiento y envejecimiento.

La tercera parte, titulada "Calidad de Vida en Argentina» consta de 6 capítulos en los cuales se fundamenta el enfoque geográfico del estudio de calidad de vida. Específicamente, en los Capítulos 8 y 9 se plantean y discuten aspectos conceptuales y metodológicos relativos a la noción de calidad de vida, estableciéndose diferencias con los conceptos de «nivel de vida" y de "pobreza». Respecto de calidad de vida, se argumenta que refiere a «una medida de logro respecto de un nivel establecido como óptimo teniendo en cuenta dimensiones socioeconómicas y ambientales dependiente de la escala de valores prevaleciente en la sociedad y que varían en función de las expectativas de progreso histórico». Una cuestión a destacar es el agrupamiento de los mencionados indicadores en dos «dimensiones» de la calidad de vida: la socioeconómica y la ambiental. La primera se encuentra específicamente referida al nivel de ingresos, la composición del grupo familiar y el capital social y educativo de los hogares. La segunda, en cambio, se asocia al acceso a la infraestructura de servicios, el entorno barrial del hogar y el hábitat. En este sentido, cabe destacar que ambas dimensiones reflejan facetas de la calidad de vida no siempre coincidentes, por lo cual su tratamiento en forma independiente ayuda a comprender más acabadamente las condiciones de vida de la población. Seguidamente, se explicita la metodología utilizada para la medición del bienestar por medio de la implementación del índice de Calidad de Vida (ICV). 
En la propuesta del libro reseñado, ambas «dimensiones» contienen a 4 "indicadores» (Educación, Salud, Vivienda e Índice de Calidad Ambiental). Estos a su vez agrupan a 9 «macrovariables» (Población de al menos is años con estudios primarios incompletos, Población con estudios universitarios completos, Tasa de Mortalidad Infantil, Población con Obra Social, Población en Viviendas sin retrete, Población con hacinamiento, Recursos recreativos de base natural, Recursos recreativos de socialmente construidos y Problemas Ambientales). Luego, 29 «variables» se implementan para dimensionar las «macrovariables». Se establece un esquema de ponderaciones y pesos diferenciales y por sumatoria se obtiene el Índice de Calidad de Vida. En general, se aplica un criterio de "cuartiles" para clasificar los resultados y producir la cartografía. Los capítulos IO, II, I2 y I3 se dedican a compilar resultados generales a nivel del país del años 2010, y de acuerdo a las comparaciones establecidas con lo obtenido para los años I99I y 200I, se evidencian notorios avances en lo que refiere a Educación, Salud y Vivienda.

La cuarta parte lleva como título «Factores de diferenciación de calidad de vida en Argentina" y agrupa a 3 capítulos. El número I4 se denomina «Calidad de vida, dinámica migratoria, centralidad/accesibilidad, categorías urbanas y PBG» y se señala que "la relación entre los niveles de calidad de vida y PBG sigue siendo clara». Agregando que «los contextos de escasa generación de riqueza resultan adversos para mejorar las condiciones de vida de la población", aunque las diferencias encontradas en 2010 habrían disminuido respecto de lo evidenciado para 200I. En cuanto a la relación entre escala urbana y calidad de vida, se ha reforzado la hipótesis de la existencia de una escala humana óptima, ya que por encima y por debajo de ella operan diferentes factores negativos. La calidad de vida más alta se ubica en «ATIs grandes» y «ATIs medias» (Vapñarsky y Gorojovsky, 1990). En el Capítulo 15: "Calidad de vida y enfermedades no transmisibles», escrito por Sonia Alejandra Pou, se sostiene que «a mejor calidad de vida en un departamento, la tasa de mortalidad por enfermedades cardiovasculares tiende a ser menor». En tanto, en el Capítulo I6, "Categorías urbanas según regiones», Guillermo Velázquez y Santiago Linares argumentan que «la región con mayor calidad ambiental es la de Cuyo, luego siguen la del NOA y el NEA. La región con peores condiciones ambientales es la metropolitana de Buenos Aires». 
La quinta parte ha sido denominada "Análisis Regional» y a través de 6 capítulos escritos por docentes-investigadores radicados en diversos centros universitarios busca aportar una mirada regional a los desbalances de la calidad de vida existentes al interior de 6 regiones del país. Un recorrido de I3I páginas permite conocer con detalle la situación de calidad de vida del noroeste, el nordeste, Cuyo, la región pampeana, la región metropolitana de Buenos Aires y la Patagonia junto a los factores relacionados, en la pluma de Fernando Longhi, Pablo Paolasso, Norma Meichrty, Alejandra Fantín, Carolina Beceyro, Rosana Castillo, Jorge Pickenhayn, Ricardo Villavicencio, Guillermo Ángel Velázquez, Néstor Javier Gómez, Claudia Baxendale, Gustavo Buzai, Jorge Morina, Gerardo De Jong y Marcos Mare.

Cartografía a color, tablas, gráficos y textos explicativos permiten al lector hacerse de una adecuada visión de la situación de calidad de vida de la Argentina regional contemporánea, con sus contradicciones intrarregionales, contrastes interregionales, el peso de las diferentes dimensiones e indicadores, los logros y las deudas.

En las «Reflexiones Finales» se señala que durante la primera década del siglo XXI los índices de calidad de vida diseñados y operacionalizados en esta obra han mejorado de manera sustancial, principalmente en lo que respecta a las dimensiones educación y salud. También se observan algunos progresos en los recursos de recreativos socialmente construidos. Aunque las mejoras tuvieron mayor peso relativo en los territorios que habían partido de situaciones relativamente más desfavorables, generándose así un proceso de "cierta convergencia entre la población residente en las diferentes regiones». Respecto de los factores de diferenciación, la dinámica migratoria no muestra mayor relación con la calidad de vida. En cambio, la centralidad y la accesibilidad continúan siendo factores consistentes. La escala urbana es otro factor insoslayable. Las ciudades intermedias serían el escenario más propicio para el bienestar.

En suma, un libro para analizar cambios, continuidades, para conocer una propuesta conceptual y metodológica que ayude a dimensionar realidades del país, de las provincias, de las ciudades y de los pueblos, pero que también colabore a pensar en nuevas propuestas a fin de seguir pensando el quehacer geográfico. 
